\title{
Global analysis of active longitudes of sunspots
}

\author{
L. Zhang ${ }^{1,2}$, K. Mursula ${ }^{1}$, I. Usoskin ${ }^{3}$, and H. Wang ${ }^{2}$ \\ 1 Department of Physics, 90014 University of Oulu, Finland \\ e-mail: kalevi .mursula@oulu.fi \\ 2 Key Laboratory of Solar Activity, National Astronomical Observatories, Chinese Academy of Sciences, Beijing, PR China \\ ${ }^{3}$ University of Oulu, Sodankylä Geophysical Observatory, Oulu, Finland
}

Received 22 June 2010 / Accepted 10 November 2010

\begin{abstract}
Context. Active longitudes have been found in various manifestations of solar activity. The longitudinal distribution of, e.g., sunspots and solar X-ray flares shows two persistent preferred longitudes separated by roughly 180 degrees. We previously studied solar X-ray flares using an improved version of a dynamic, differentially rotating coordinate system and found enhanced rotational asymmetry and rotation parameter values that are consistent for the three classes of X-ray flares.

Aims. We aim to find the optimal values of rotation parameters of active longitudes of sunspots for several different time intervals and separately for the two solar hemispheres.

Methods. We perform a global study of the longitudinal location of sunspots (all sunspots and first appearance sunspots) using a refined version of a dynamic, differentially rotating coordinate system.

Results. We find that the rotation parameters for sunspots are in good agreement with those obtained for X-ray flares using the same method. The improved method typically finds somewhat faster equatorial rotation with better accuracy. The improved treatment also leads to a larger non-axisymmetry. Rotation parameters for all sunspots and first appearances closely agree with each other, but nonaxisymmetry is systematically larger for all sunspots than for first appearances, suggesting that strong fields follow more closely the pattern of active longitudes. The refined method emphasizes hemispheric differences in rotation. Over the whole interval, the mean rotation in the southern hemisphere is slower than in the north. We also find significant temporal variability in the two rotation parameters over the 136-year interval. Interestingly, the long-term variations (trends and residual oscillations) in solar rotation are roughly the opposite in the northern and southern hemispheres.

Conclusions. Rotation parameters vary differently with time in the northern and southern hemispheres. Both sunspots and flares strongly suggest that the northern hemisphere rotated considerably faster but the southern hemisphere slightly slower than the Carrington rotation rate during the last three solar cycles.
\end{abstract}

Key words. Sun: rotation - sunspots

\section{Introduction}

Active longitudes of the Sun have been studied in detail since the early 20th century (Chidambara 1932; Losh 1939). It has been found that various manifestations of solar activity, such as sunspots (Berdyugina et al. 2003; Usoskin et al. 2005; Juckett 2006), solar magnetic field (Benevolenskaya et al. 1999; Bumba et al. 2000), heliospheric magnetic field (Ruzmaikin et al. 2001; Takalo \& Mursula 2002; Mursula \& Hiltula 2004), and flares (Bai 1988, 2003) occur preferentially at specific longitudes that are called active longitudes. However, lifetimes and rotation velocities of active longitudes obtained for different tracers of solar activity have been found to vary significantly. Balthasar (2007) noted, by analyzing sunspot numbers (1818-2006), that the rotation period varies over long timescales. Heristchi \& Mouradian (2009) found an increase in the rotation rate from cycle 19 to cycle 23 by calculating sunspot numbers and the coronal radio flux at $10.7 \mathrm{~cm}$. Pelt et al. (2010), using a nonparametric statistical analysis of the longitude of sunspot groups in 1874-2008, proposed that the strong tendency for sunspot groups to cluster at a certain longitude peters out with time, the longest observable correlations reaching 10-15 Carrington rotations. This result, however, is more relevant for active regions than for the long-living active longitudes studied here.
Using a dynamic, differentially rotating coordinate system, Usoskin et al. (2005) found that two active longitudes of sunspots separated by about $180^{\circ}$ existed for the whole time interval of sunspots with measured locations (1878-1996). The same system was used to analyze the longitudinal occurrence of solar X-ray flares observed by the NOAA/GOES satellites (Zhang et al. 2007a,b). However, in these studies the differential rotation parameters for sunspots were quite different from those obtained for X-ray flares. Even the parameters for the different X-ray class flares when calculated separately were found to be quite different (Zhang et al. 2007b).

We have recently conducted a global search of the optimized rotation parameters for the three classes of solar X-ray flares using a corrected version of the dynamic, differentially rotating coordinate system (Zhang et al. 2011). We found that the refined parameters describing the rotation of active longitudes are mutually consistent for the three flare classes and that the northern hemisphere rotates significantly faster than the southern hemisphere during the last three solar cycles. This contradicts the result of Heristchi \& Mouradian (2009), who found no significant difference between the rotation rates of the two hemispheres. Moreover, the refined parameters yielded a consistently higher level of non-axisymmetry for the longitudinal distribution, thus increasing evidence for the existence of active longitudes. 
Here we perform a global search to find the refined optimal values of parameters describing the longitudinal distribution of sunspots, all sunspots and the first appearance of sunspots separately, using the corrected version of the dynamic, differentially rotating coordinate system. We obtain the global best-fit values of parameters describing the differential rotation of active longitudes of sunspots, and estimate their statistical errors and the level of non-axisymmetry. We compare these values for sunspots with the corresponding values of parameters found for solar X-ray flares.

The paper is organized as follows. The data is introduced in Sect. 2. The analysis method based on the corrected version of the dynamic, differentially rotating coordinate system is described in Sect. 3. The results obtained for the differential rotation parameters and non-axisymmetry are presented in Sects. 4 and 5. The results are discussed in Sect. 6. In Sect. 7 we present the final conclusions of this study.

\section{Data}

We use here the sunspot group data collected by the Royal Greenwich Observatory and the USAF/NOAA for the years 1874-2009 (http://solarscience.msfc.nasa.gov/ greenwch. shtml). We use this data of sunspot group locations and areas in two different ways. Firstly, only the first appearance of each sunspot group is taken into account, i.e., each sunspot group is considered only once when it is first mentioned in the data (either on the day of its birth or when it first appeared in the East limb), ignoring all later records of the spot. It is important to study the newly appeared sunspots because they are considered to better indicate the emergence of magnetic flux through the photosphere, which is a key process of the solar cycle (Solanki 2003) and of the study of the solar magnetic dynamo. Secondly, all daily records of sunspots are taken into account, irrespective of whether they are new or old. We also note that we use here data that are uncorrected for the change in data source in 1977 and ignore the area correction factor of 1.4 since 1977 (see above web page). However, this change in the homogeneity of the database has little importance for the type of studies conducted here.

\section{Improved analysis method}

Differential rotation of the solar surface can be described approximately as

$\Omega_{\phi}=\Omega_{0}-B \sin ^{2} \phi$,

where $\Omega_{\phi}$ (deg/day) is the sidereal angular velocity at a given latitude $\phi, \Omega_{0}$ (deg/day) is the (sidereal) equatorial angular velocity and $B$ (deg/day) describes the differential rotation rate. Rotation parameters $\Omega_{0}$ and $B$ are constants whose values will be determined by optimization.

Taking the Carrington rotation period as the time step, the normalized area of a $k$ th sunspot group in the $j$ th rotation is

$W_{j k}=A_{j k} / \sum_{k} A_{j k}$

where $A_{j k}$ is the observed sunspot area (corrected for projection), and the sum is taken over all spots in the $j$ th rotation. The sunspot area-weighted latitude for this rotation is then

$\left\langle\phi_{j}\right\rangle=\sum_{k} W_{j k} \phi_{j k}$ where $\phi_{j k}$ denotes the observed latitude of the $k$ th spot in the $j$ th rotation. For Carrington rotations with no sunspots, we use the linear interpolation of the mean latitude. Accordingly, the (sidereal) angular velocity for the $j$ th rotation is

$\Omega_{N_{j}}=\Omega_{0}-B \sin ^{2}\left\langle\phi_{j}\right\rangle$.

On the $k$ th day of the $i$ th Carrington rotation, the migration $M$ of an active longitude with respect to the Carrington reference frame is determined by

$M=T_{\mathrm{c}} \sum_{j=N_{0}}^{N_{i-1}}\left(\Omega_{j}-\Omega_{\mathrm{c}}\right)+k\left(\Omega_{N_{i}}-\Omega_{\mathrm{c}}\right)$,

where $N_{0}$ and $N_{i-1}$ denote the Carrington rotation numbers of the first and the $(i-1)$ th rotation of the data set, $T_{\mathrm{c}}=27.2753$ days is the synodic Carrington rotation period, $\Omega_{\mathrm{c}}$ is the angular velocity of Carrington frame (in sidereal frame $14.1844 \mathrm{deg} /$ day and in synodic frame $13.199 \mathrm{deg} / \mathrm{day}$ ), and $k$ is the time of sunspot group observation given as a fractional day of the Carrington rotation.

Let us assume that one active longitude in the beginning is at Carrington longitude $\Lambda_{01}$. It will reach $\Lambda_{i k 1}$ on the $k$ th day of the $i$ th Carrington rotation, which can be expressed as

$\Lambda_{i k 1}=\left(\Lambda_{01}+M\right) \bmod 360^{\circ}$,

where $\bmod 360^{\circ}$ means modulo $360^{\circ}$ within the range $\left[0^{\circ}, 360^{\circ}\right]$. The other active longitude is assumed to be at $\Lambda_{i k 2}=\Lambda_{i k 1} \pm 180^{\circ}$, i.e., opposite to $\Lambda_{i k 1}$.

The deviation $\Delta_{i k}$ between the longitudinal position $\lambda_{i k}$ of a sunspot, which occurred on the $k$ th day in the ith rotation, and one of the two active longitude bands $\left(\Lambda_{i k 1}\right.$ or $\left.\Lambda_{i k 2}\right)$ is

$\Delta_{i k}=\min \left(\left|\lambda_{i k}-\Lambda_{i k 1}\right|, 360^{\circ}-\left|\lambda_{i k}-\Lambda_{i k 1}\right|,\left|180^{\circ}-\right| \lambda_{i k}-\Lambda_{i k 1}||\right)$.

In order to illustrate this, we discuss some examples. Assuming $\Lambda_{i k 1}=2^{\circ}$ and $\Lambda_{i k 2}=182^{\circ}$, and taking $\lambda_{i k}=5^{\circ}$, one obtains $\Delta_{i k}=$ $\left|\lambda_{i k}-\Lambda_{i k 1}\right|=3^{\circ}$. On the other hand, taking $\lambda_{i k}=359^{\circ}$, yields correspondingly $\Delta_{i k}=360^{\circ}-\left|\lambda_{i k}-\Lambda_{i k 1}\right|=3^{\circ}$. When $\lambda_{i k}=179^{\circ}$ or $\lambda_{i k}=185^{\circ}, \Delta_{i k}=\left|180^{\circ}-\right| \lambda_{i k}-\Lambda_{i k 1}\left|=3^{\circ}\right|$.

We note that the first studies using the dynamic reference frame (Usoskin et al. 2005; Zhang et al. 2007b) missed the second term on the right-hand side of Eq. (5). Thus, the mean migration with respect to the Carrington rotation (the first term on the right-hand side of Eq. (5)) was used as the migration of active longitudes from the beginning to the end of the rotation, which resulted in only one active longitude $\Lambda_{i 1}$ in the $i$ th rotation being used for each spot of that rotation when defining the deviation for spots according to Eq. (7). Adding the second term of Eq. (5), we obtain a more accurate, smoothly changing active longitude $\Lambda_{i k 1}$ at the time when a spot occurs, and a more precise deviation $\Delta_{i k}$ in Eq. (7) by calculating $\left|\lambda_{i k}-\Lambda_{i k 1}\right|$, rather than $\left|\lambda_{i k}-\Lambda_{i 1}\right|$. This improved recipe was first adopted in a recent study of the active longitudes of solar X-ray flares (Zhang et al. 2011).

We define the merit function (which is to be minimized) as the weighted mean squared deviation of sunspots from the nearest active longitude

$\epsilon=\frac{1}{n} \sum_{i} \sum_{k} W_{j k} \Delta_{i k}^{2}$,

where $\mathrm{n}$ denotes the total number of sunspots and $W_{j k}$ is defined in Eq. (2). We first perform a global search of the bestfit parameters as the global minimum of the merit function by 

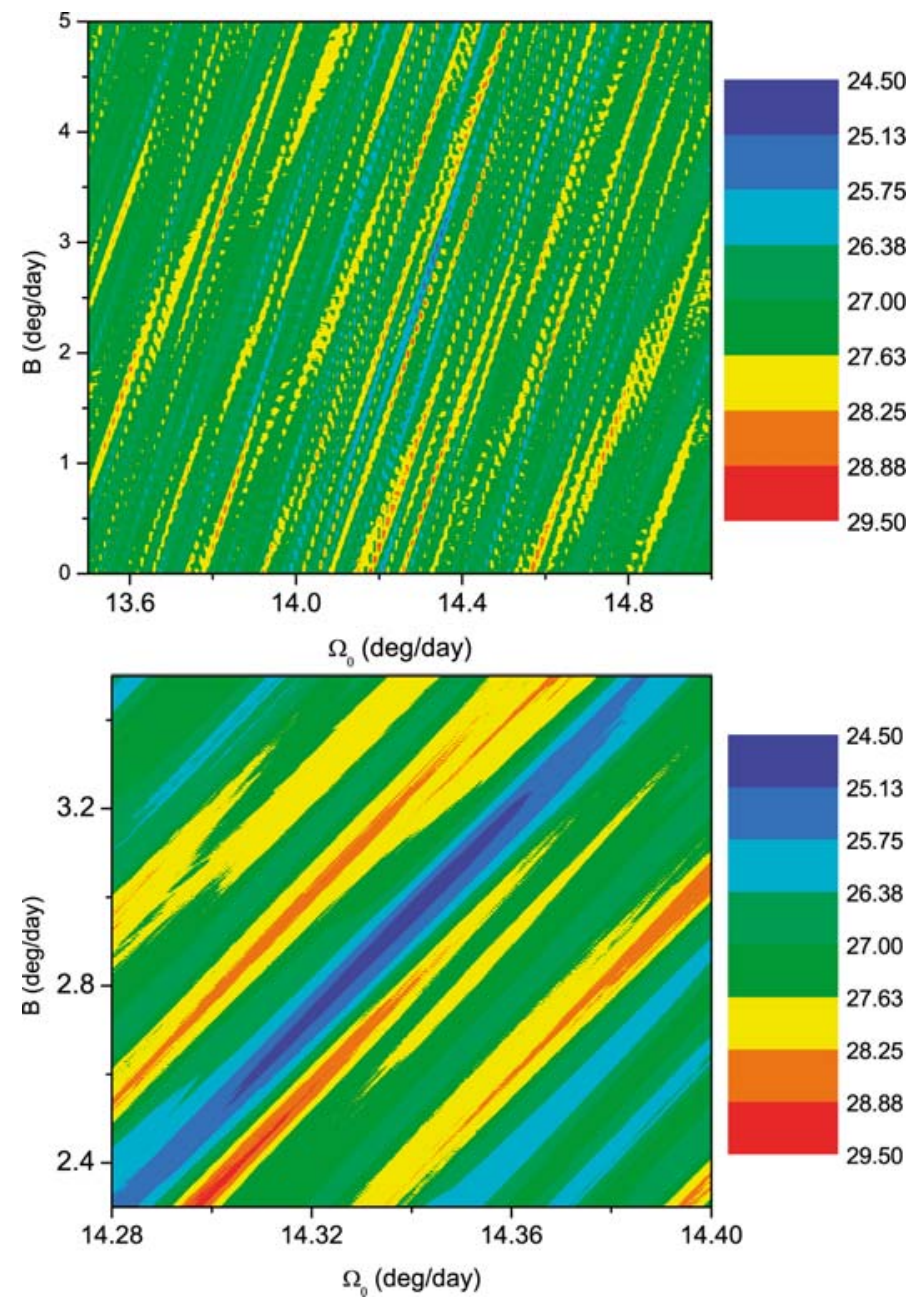

Fig. 1. Values of the merit function in color coding (in units of $\mathrm{deg}^{2}$ ) as a function of $\Omega_{0}$ and $B$ for all sunspots of the southern hemisphere in the last three solar cycles (a, top) over the full studied parameter range; $(\mathbf{b}$, bottom $)$ in the limited region around the best-fit parameter values.

varying the value of $\Omega_{0}$ in the interval $[13.5,15.0]$ (deg/day) in steps of 0.01 (deg/day), $B$ within $[0.0,5.0]$ (deg/day) in steps of 0.01 (deg/day) and $\Lambda_{01}$ within $\left[0^{\circ}, 360^{\circ}\right]$ in steps of $1^{\circ}$. After finding the global minimum, we perform another round of optimization over a more limited parameter range using steps of 0.001 (deg/day) for both $\Omega_{0}$ and $B$. We always analyze the sunspots in the northern and southern hemispheres separately. We also define all parameters for two data sets, one using all sunspot groups included in the above mentioned database and the other using the first appearance of spots only. We also use different time intervals, varying the length of intervals from one solar cycle to three cycles and six cycles, as well as using the 129-year interval (1878-1996) of Usoskin et al. (2005) and the whole 136-year period of data in 1874-2009.

Figure 1 shows the values of the merit function in color coding as a function of $\Omega_{0}$ and $B$ for all sunspots in the southern hemisphere during the last three solar cycles. Figure 1a depicts the global situation over the whole parameter range, showing the global minimum of the merit function as the only region where the lowest values (depicted by dark blue color) are found. The striped structure of the merit function in Fig. 1a (and all similar plots) is a result of the correlation of the two parameters and the different sensitivity of the merit function to them. Figure $1 \mathrm{~b}$ shows the detailed situation of the merit function in a more limited range (the area corresponding to the smallest bin value of merit function in Fig. 1a) around the best-fit parameters found in the global search, using the smaller step size of 0.001 (deg/day) for $\Omega_{0}$ and $B$. Finding the minimum of the merit function in the reduced range by using a finer step size we can calculate the bestfit parameters more accurately. Moreover, detailed plots such as Fig. 1b were used to obtain the errors in the two parameters. As depicted in Fig. 1b, the values of the merit function were divided to eight equal bins between the largest and the smallest value within the range included in Fig. 1b. The parameter errors were then calculated as corresponding to the standard deviations of the merit function in either direction within the region of its smallest value (denoted in dark blue in Fig. 1b).

\section{Differential rotation parameters}

We have listed the optimal values of the differential rotation parameters with error bars for the different time intervals in Tables $1-4$. In addition to the parameters $\Omega_{0}$, these tables also include the values of $\Omega_{17}$, the rotation angular velocity at the latitude of $17^{\circ}$, which is the mean latitude of sunspots. In each table we have treated the two hemispheres separately and calculated the optimal parameters both using all sunspots and the first appearances of spots. Table 1 gives the parameter values and their errors for each solar cycle separately. Table 2 lists the same parameters for three-cycle time intervals and Table 3 for six-cycle intervals. Finally, Table 4 depicts the parameters for the whole time interval 1974-2009, as well as for 1874-1996, allowing comparison with the earlier results (Usoskin et al. 2005).

Figure 2 shows the merit function for a detailed analysis of all sunspots observed in the southern hemisphere during solar cycle 21 (Fig. 2a), during the last six solar cycles (Fig. 2b) and for the whole 136-year interval (Fig. 2c). Obviously, both the value and the accuracy of the fit parameters depend on the length of the analyzed period, with less accurate values obtained for shorter lengths. For solar cycle 21 (Fig. 2a), the optimal equatorial angular velocity (see Table 1 ) is $\Omega_{0}=14.240 \pm$ 0.018 (deg/day), while for the last six solar cycles, solar cycles $18-23$ (see Table 3 ) it is $\Omega_{0}=14.322 \pm 0.011(\mathrm{deg} / \mathrm{day}$ ) and for the whole interval $\Omega_{0}=14.401 \pm 0.004$ (deg/day). The improved accuracy in the two parameters is seen as the narrowing and shortening of the best-fit region in Fig. 2. However, we note that the accuracy does not improve very quickly when increasing the time interval from one cycle to six cycles or 136 years because the different cycles rotate slightly differently. The average error bars of the best-fit parameters $\Omega_{0}, B$, and $\Omega_{17}$ denoted by $E_{\Omega_{0}}, E_{B}$, and $E_{\Omega_{17}}$ are given in Table 5 for the different time intervals. The average errors decrease slowly but systematically with increasing time interval. For instance, the mean error bars of $\Omega_{17}$ for the first appearance of spots decrease from \pm 0.005 (deg/day) for 1 -cycle values to \pm 0.001 (deg/day) for 136 -year values in both hemispheres. The same decrease is seen for all parameters and both hemispheres.

In Fig. 3 we have depicted the values of $\Omega_{0}$ using all sunspots for the individual cycles and three-cycle intervals (from Tables 1 and 2). The two upper (lower) panels show the results for the northern (southern) hemisphere, while the left panels depict the individual cycles, and the right panels the three-cycle periods. It is interesting to see how much the optimal values of $\Omega_{0}$ vary among the individual cycles and even between the two hemispheres of one cycle. The fastest equatorial rotation is seen during cycle 14 and the slowest in cycle 22, both in the southern hemisphere. The slowest and fastest equatorial rotations in the northern hemisphere are seen during the successive cycles 20 
Table 1. Rotation parameters for individual cycles in units of deg/day.

\begin{tabular}{|c|c|c|c|c|c|c|}
\hline & \multicolumn{6}{|c|}{ Northern hemisphere } \\
\hline & \multicolumn{3}{|c|}{ First appearance of spots } & \multicolumn{3}{|c|}{ All spots } \\
\hline cycle & $\Omega_{0}$ & $B$ & $\Omega_{17}$ & $\Omega_{0}$ & $B$ & $\Omega_{17}$ \\
\hline 12 & $14.280 \pm 0.030$ & $1.547 \pm 0.372$ & $14.151 \pm 0.006$ & $14.283 \pm 0.010$ & $1.505 \pm 0.133$ & $14.153 \pm 0.003$ \\
\hline 13 & $14.209 \pm 0.017$ & $3.497 \pm 0.241$ & $13.910 \pm 0.005$ & $14.200 \pm 0.016$ & $3.319 \pm 0.243$ & $13.915 \pm 0.005$ \\
\hline 14 & $14.226 \pm 0.021$ & $1.166 \pm 0.245$ & $14.125 \pm 0.004$ & $14.242 \pm 0.016$ & $1.664 \pm 0.241$ & $14.100 \pm 0.005$ \\
\hline 15 & $14.387 \pm 0.011$ & $3.828 \pm 0.153$ & $14.060 \pm 0.003$ & $14.346 \pm 0.012$ & $3.021 \pm 0.172$ & $14.086 \pm 0.003$ \\
\hline 16 & $14.429 \pm 0.017$ & $3.726 \pm 0.204$ & $14.110 \pm 0.004$ & $14.433 \pm 0.020$ & $3.827 \pm 0.236$ & $14.106 \pm 0.004$ \\
\hline 17 & $14.431 \pm 0.033$ & $4.223 \pm 0.412$ & $14.068 \pm 0.003$ & $14.437 \pm 0.007$ & $4.033 \pm 0.077$ & $14.092 \pm 0.001$ \\
\hline 18 & $14.369 \pm 0.022$ & $0.406 \pm 0.260$ & $14.331 \pm 0.004$ & $14.374 \pm 0.015$ & $0.467 \pm 0.163$ & $14.331 \pm 0.003$ \\
\hline 19 & $14.309 \pm 0.016$ & $1.524 \pm 0.156$ & $14.179 \pm 0.004$ & $14.292 \pm 0.016$ & $1.347 \pm 0.158$ & $14.177 \pm 0.003$ \\
\hline 20 & $14.203 \pm 0.015$ & $2.515 \pm 0.200$ & $13.988 \pm 0.003$ & $14.188 \pm 0.009$ & $2.340 \pm 0.115$ & $13.988 \pm 0.002$ \\
\hline 21 & $14.595 \pm 0.030$ & $3.435 \pm 0.357$ & $14.302 \pm 0.004$ & $14.599 \pm 0.025$ & $3.780 \pm 0.289$ & $14.273 \pm 0.003$ \\
\hline 22 & $14.495 \pm 0.010$ & $2.162 \pm 0.098$ & $14.309 \pm 0.002$ & $14.497 \pm 0.024$ & $2.090 \pm 0.254$ & $14.318 \pm 0.005$ \\
\hline \multirow[t]{3}{*}{23} & $14.511 \pm 0.031$ & $0.421 \pm 0.481$ & $14.467 \pm 0.010$ & $14.530 \pm 0.034$ & $0.659 \pm 0.448$ & $14.471 \pm 0.008$ \\
\hline & \multicolumn{6}{|c|}{ Southern hemisphere } \\
\hline & \multicolumn{3}{|c|}{ First appearance of spots } & \multicolumn{3}{|c|}{ All spots } \\
\hline cycle & $\Omega_{0}$ & $\bar{B}$ & $\Omega_{17}$ & $\Omega_{0}$ & $\bar{B}$ & $\Omega_{17}$ \\
\hline 12 & $14.348 \pm 0.016$ & $3.004 \pm 0.209$ & $14.092 \pm 0.003$ & $14.337 \pm 0.015$ & $2.975 \pm 0.200$ & $14.081 \pm 0.003$ \\
\hline 13 & $14.430 \pm 0.024$ & $1.008 \pm 0.277$ & $14.344 \pm 0.008$ & $14.436 \pm 0.015$ & $1.147 \pm 0.199$ & $14.338 \pm 0.002$ \\
\hline 14 & $14.536 \pm 0.014$ & $2.724 \pm 0.219$ & $14.302 \pm 0.005$ & $14.606 \pm 0.017$ & $2.927 \pm 0.275$ & $14.353 \pm 0.006$ \\
\hline 15 & $14.485 \pm 0.017$ & $1.023 \pm 0.209$ & $14.398 \pm 0.003$ & $14.489 \pm 0.022$ & $1.277 \pm 0.271$ & $14.380 \pm 0.003$ \\
\hline 16 & $14.550 \pm 0.011$ & $2.630 \pm 0.123$ & $14.325 \pm 0.003$ & $14.549 \pm 0.009$ & $2.640 \pm 0.109$ & $14.323 \pm 0.002$ \\
\hline 17 & $14.288 \pm 0.020$ & $4.578 \pm 0.214$ & $13.899 \pm 0.004$ & $14.300 \pm 0.011$ & $4.680 \pm 0.112$ & $13.899 \pm 0.003$ \\
\hline 18 & $14.241 \pm 0.017$ & $3.924 \pm 0.185$ & $13.907 \pm 0.003$ & $14.229 \pm 0.020$ & $3.707 \pm 0.201$ & $13.912 \pm 0.004$ \\
\hline 19 & $14.217 \pm 0.026$ & $4.252 \pm 0.231$ & $13.849 \pm 0.007$ & $14.207 \pm 0.017$ & $4.044 \pm 0.185$ & $13.859 \pm 0.003$ \\
\hline 20 & $14.232 \pm 0.027$ & $2.646 \pm 0.335$ & $14.006 \pm 0.005$ & $14.254 \pm 0.012$ & $3.096 \pm 0.141$ & $13.989 \pm 0.003$ \\
\hline 21 & $14.236 \pm 0.027$ & $1.926 \pm 0.293$ & $14.071 \pm 0.004$ & $14.240 \pm 0.018$ & $2.010 \pm 0.202$ & $14.068 \pm 0.003$ \\
\hline 22 & $14.190 \pm 0.027$ & $1.420 \pm 0.235$ & $14.068 \pm 0.007$ & $14.158 \pm 0.015$ & $1.270 \pm 0.121$ & $14.055 \pm 0.005$ \\
\hline 23 & $14.460 \pm 0.026$ & $0.901 \pm 0.309$ & $14.380 \pm 0.008$ & $14.461 \pm 0.024$ & $0.912 \pm 0.293$ & $14.383 \pm 0.003$ \\
\hline
\end{tabular}

Table 2. Rotation parameters for three-cycle intervals in units of deg/day.

\begin{tabular}{|c|c|c|c|c|c|c|}
\hline & \multicolumn{6}{|c|}{ Northern hemisphere } \\
\hline & \multicolumn{3}{|c|}{ First appearance of spots } & \multicolumn{3}{|c|}{ All spots } \\
\hline cycle & $\Omega_{0}$ & $B$ & $\Omega_{17}$ & $\overline{\Omega_{0}}$ & B & $\Omega_{17}$ \\
\hline $12-14$ & $14.221 \pm 0.023$ & $4.014 \pm 0.301$ & $13.877 \pm 0.007$ & $14.206 \pm 0.015$ & $4.248 \pm 0.237$ & $13.846 \pm 0.005$ \\
\hline $15-17$ & $14.381 \pm 0.022$ & $3.583 \pm 0.297$ & $14.078 \pm 0.004$ & $14.380 \pm 0.019$ & $3.516 \pm 0.274$ & $14.081 \pm 0.004$ \\
\hline $18-20$ & $14.414 \pm 0.015$ & $3.516 \pm 0.182$ & $14.114 \pm 0.001$ & $14.426 \pm 0.014$ & $3.596 \pm 0.168$ & $14.117 \pm 0.001$ \\
\hline \multirow[t]{3}{*}{$21-23$} & $14.579 \pm 0.021$ & $1.488 \pm 0.260$ & $14.452 \pm 0.003$ & $14.566 \pm 0.019$ & $1.322 \pm 0.252$ & $14.453 \pm 0.003$ \\
\hline & \multicolumn{6}{|c|}{ Southern hemisphere } \\
\hline & \multicolumn{3}{|c|}{ First appearance of spots } & \multicolumn{3}{|c|}{ All spots } \\
\hline cycle & $\Omega_{0}$ & $B$ & $\Omega_{17}$ & $\Omega_{0}$ & $B$ & $\Omega_{17}$ \\
\hline $12-14$ & $14.372 \pm 0.017$ & $1.035 \pm 0.240$ & $14.284 \pm 0.004$ & $14.344 \pm 0.020$ & $0.604 \pm 0.281$ & $14.292 \pm 0.004$ \\
\hline $15-17$ & $14.585 \pm 0.020$ & $3.168 \pm 0.268$ & $14.315 \pm 0.003$ & $14.571 \pm 0.018$ & $3.041 \pm 0.243$ & $14.311 \pm 0.002$ \\
\hline $18-20$ & $14.513 \pm 0.018$ & $4.058 \pm 0.252$ & $14.168 \pm 0.005$ & $14.527 \pm 0.009$ & $4.517 \pm 0.110$ & $14.141 \pm 0.001$ \\
\hline $21-23$ & $14.352 \pm 0.007$ & $3.068 \pm 0.084$ & $14.089 \pm 0.001$ & $14.337 \pm 0.011$ & $2.936 \pm 0.135$ & $14.087 \pm 0.001$ \\
\hline
\end{tabular}

Table 3. Rotation parameters for six-cycle intervals in units of deg/day.

\begin{tabular}{|c|c|c|c|c|c|c|}
\hline & \multicolumn{6}{|c|}{ Northern hemisphere } \\
\hline & \multicolumn{4}{|c|}{ First appearance of spots } & \multicolumn{2}{|l|}{ All spots } \\
\hline cycle & $\Omega_{0}$ & $B$ & $\Omega_{17}$ & $\Omega_{0}$ & $B$ & $\Omega_{17}$ \\
\hline $12-17$ & $14.355 \pm 0.012$ & $3.116 \pm 0.198$ & $14.089 \pm 0.004$ & $14.305 \pm 0.010$ & $2.496 \pm 0.171$ & $14.091 \pm 0.003$ \\
\hline $18-23$ & $14.588 \pm 0.015$ & $3.331 \pm 0.196$ & $14.303 \pm 0.002$ & $14.623 \pm 0.009$ & $2.687 \pm 0.109$ & $14.393 \pm 0.001$ \\
\hline & \multicolumn{6}{|c|}{ Southern hemisphere } \\
\hline $\begin{array}{l}\text { cycle } \\
12-17 \\
18-23\end{array}$ & $\begin{array}{c}\Omega_{0} \\
14.611 \pm 0.011 \\
14.347 \pm 0.014\end{array}$ & $\begin{array}{c}B \\
3.399 \pm 0.141 \\
2.939 \pm 0.193\end{array}$ & $\begin{array}{c}\Omega_{17} \\
14.3107 \pm 0.002 \\
14.095 \pm 0.002\end{array}$ & $\begin{array}{c}\Omega_{0} \\
14.614 \pm 0.011 \\
14.322 \pm 0.011\end{array}$ & $\begin{array}{c}B \\
3.608 \pm 0.161 \\
2.806 \pm 0.150\end{array}$ & $\begin{array}{c}\Omega_{17} \\
14.305 \pm 0.003 \\
14.072 \pm 0.001\end{array}$ \\
\hline
\end{tabular}


Table 4. Rotation parameters for 1878-1996 and 1874-2009 in units of deg/day.

\begin{tabular}{ccccccc}
\hline \hline \multicolumn{6}{c}{ First appearance of spots } & \multicolumn{3}{c}{ Northern hemisphere } & All spots \\
\hline time & $\Omega_{0}$ & $B$ & $\Omega_{17}$ & $\Omega_{0}$ & $B$ & $\Omega_{17}$ \\
$1878-1996$ & $14.393 \pm 0.008$ & $3.309 \pm 0.116$ & $14.119 \pm 0.003$ & $14.414 \pm 0.007$ & $3.509 \pm 0.119$ & $14.114 \pm 0.002$ \\
$1874-2009$ & $14.424 \pm 0.005$ & $3.680 \pm 0.072$ & $14.111 \pm 0.001$ & $14.411 \pm 0.003$ & $3.455 \pm 0.050$ & $14.115 \pm 0.001$ \\
\hline \multicolumn{8}{c}{ First appearance of spots } & \multicolumn{3}{c}{ All spots } & \\
\hline time & $\Omega_{0}$ & $\Omega_{17}$ & $\Omega_{0}$ & $B$ & $\Omega_{17}$ \\
$1878-1996$ & $14.435 \pm 0.008$ & $4.931 \pm 0.113$ & $14.008 \pm 0.002$ & $14.413 \pm 0.004$ & $4.792 \pm 0.067$ & $14.003 \pm 0.001$ \\
$1874-2009$ & $14.420 \pm 0.005$ & $4.855 \pm 0.073$ & $14.006 \pm 0.001$ & $14.401 \pm 0.004$ & $4.652 \pm 0.083$ & $14.006 \pm 0.001$ \\
\hline
\end{tabular}
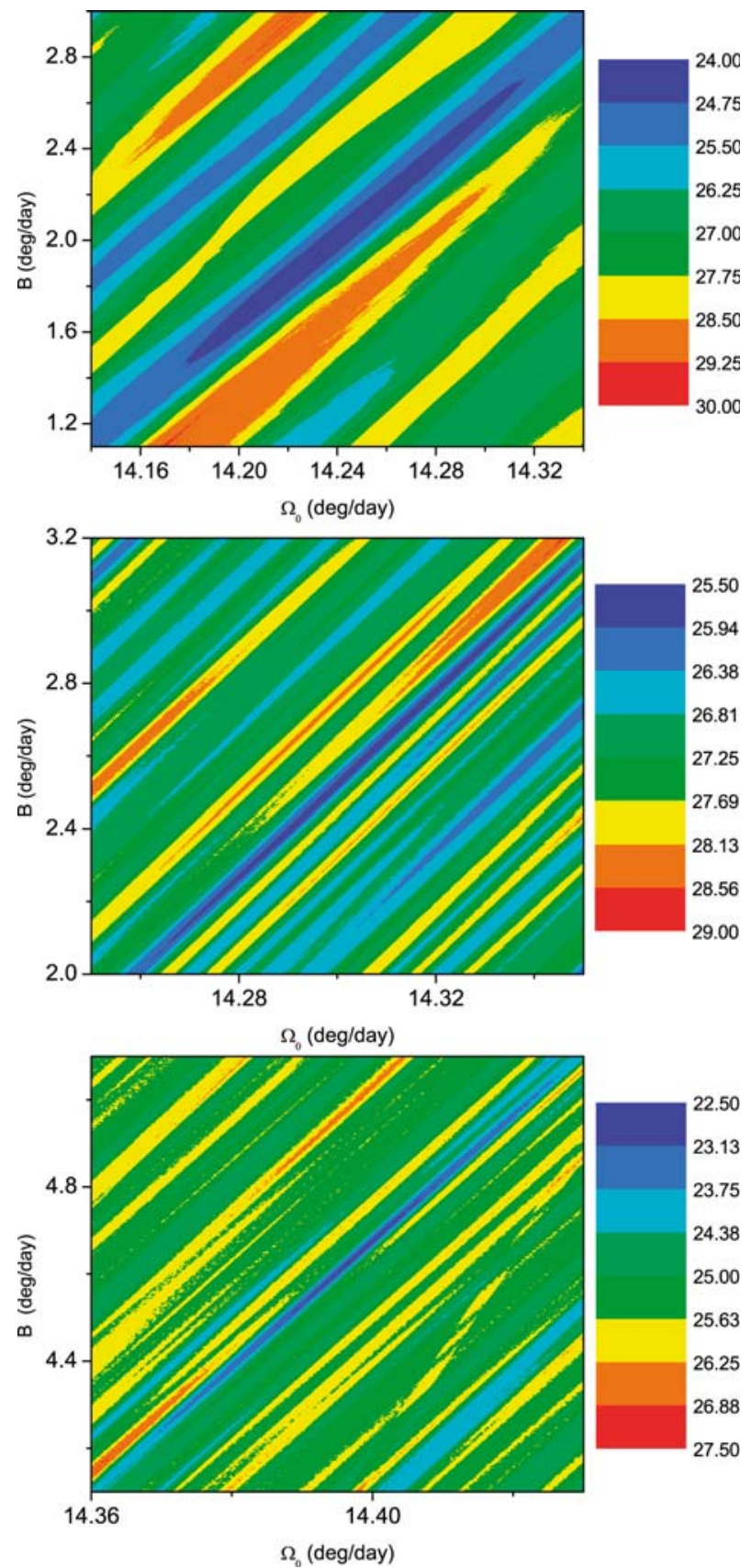

Fig. 2. Values of the merit function in color coding (in units of $\mathrm{deg}^{2}$ ) as a function of $\Omega_{0}$ and $B$ for all sunspots of the southern hemisphere in the limited region around the best-fit parameter values (a, top) for solar cycle 21; (b, center) for cycles 18-23; (c, bottom) for 1874-2009. and 21 , indicating the largest change in the $\Omega_{0}$ value between two solar cycles.

The three-cycle $\Omega_{0}$ values are mostly quite close to the average value of the corresponding three individual cycles. We note that this does not necessarily have to be so because the individual cycle fitting includes two more starting longitudes as additional free parameters. However, that the 1-cycle and three-cycle values agree with each other provides additional evidence of the robustness of the method and the long-term persistence of the active longitudes. Only one three-cycle average for cycles 18 20 deviates from the corresponding individual cycles. This could mean that a temporary phase shift may occur within this time. Interestingly, we also find (see Fig. 6) that the asymmetries for the individual cycles are lowest for these cycles.

Table 1 shows that the $\Omega_{0}$ values for the firstly appeared sunspots are very close to those obtained for all sunspots. Except for the north of cycle 15 and for the south of cycles 14 and 22, they agree within the errors for all cycles and hemispheres. This close agreement is perhaps slightly surprising, but shows that the evolution of active longitudes is rather insensitive to how the sunspots are classified. Actually, in the database used, not all first appearances are new sunspots (because some were already borne on the invisible side) or firstly appeared sunspots (because some spots lived long enough to reappear). In addition, most sunspots do not live very long, experiencing only a small phase change due to their specific motion. These features alleviate any existing differences between the two sunspot classes. Table 5 shows that the parameters for all sunspots have slightly smaller average errors than the firstly appeared spots. This is particularly true for the basic fit parameters $\Omega_{0}$ and $B$, but the errors of $\Omega_{17}$ approach each other for long time intervals.

Figure 4 depicts the optimal $B$ parameters values using all sunspots for all individual cycles. The $B$ values vary greatly from cycle to cycle, relatively much more than the $\Omega_{0}$ parameters, attaining the lowest value of 0.467 during cycle 18 in the northern hemisphere and the largest value of 4.68 in cycle 17 in the south. The $B$ values for firstly appeared sunspots agree within the error bars with those for all spots except for the north of cycle 15 where they differ by three standard deviations. This good agreement gives support that, despite their rather larger range, the optimal $B$ values are reliable. We also note that for both hemispheres the $B$ values calculated for the whole 136-year interval (see Table 4) are clearly above the mean $B$ value for individual cycles. This is another demonstration that the optimal parameter values can, for the reasons discussed above, slightly vary depending on the time interval studied. Finally, Fig. 5 depicts the values of $\Omega_{17}$ calculated using Eq. (1) and the optimal values for $\Omega_{0}$ and $B$. The panels are for the same cases as in Fig. 3 for $\Omega_{0}$. 
A\&A 529, A23 (2011)
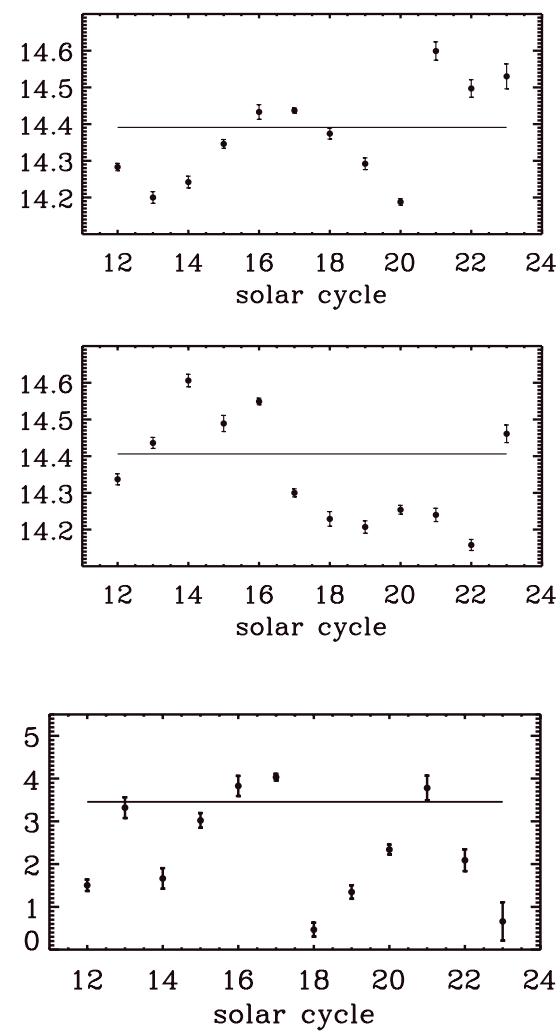
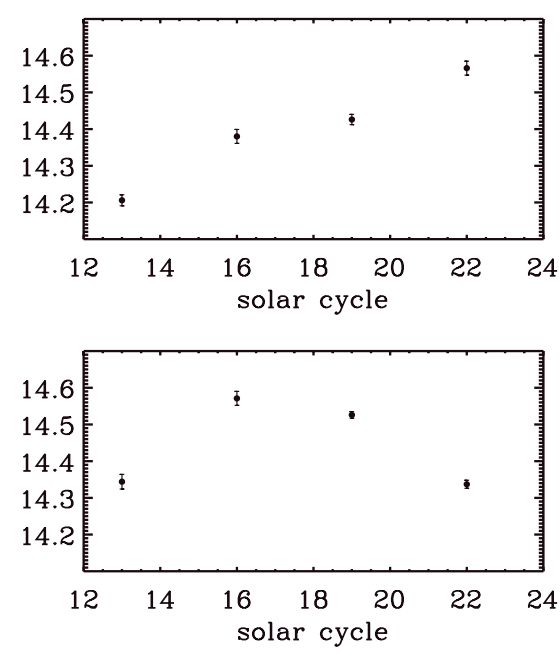

Fig. 3. Values of $\Omega_{0}$ using all sunspots for (left panels) individual cycles and (right panels) three-cycle intervals. The upper (lower) panels are for the northern (southern) hemisphere. The horizontal lines depict the best-fit value of $\Omega_{0}$ for the whole interval.

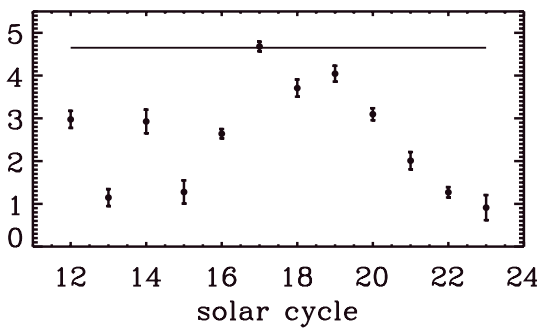
sunspots for (left) the northern and (right) southern hemisphere of all individual cycles. The horizontal lines depict the best-fit values of $B$ for the whole interval.
Fig. 4. Values of the $B$ parameter using all

Table 5. Average errors for different time intervals in units of deg/day.

\begin{tabular}{|c|c|c|c|c|c|c|}
\hline & \multicolumn{6}{|c|}{ Northern hemisphere } \\
\hline & \multicolumn{3}{|c|}{ First appearance of spots } & \multicolumn{3}{|c|}{ All spots } \\
\hline & $E_{\Omega_{0}}$ & $E_{B}$ & $\overline{E_{\Omega_{17}}}$ & $E_{\Omega_{0}}$ & $\overline{E_{B}}$ & $E_{\Omega_{17}}$ \\
\hline 1 cycle & \pm 0.021 & \pm 0.265 & \pm 0.005 & \pm 0.017 & \pm 0.210 & \pm 0.004 \\
\hline $3-$ сус & 0 & \pm & \pm 0.004 & $0 .($ & 5 & $=0$ \\
\hline 6-cycle & .0 & \pm( & \pm 0.003 & \pm 0. & \pm 0 & $=0$. \\
\hline $1878-1996$ & $=0.009$ & \pm 0.1 & \pm 0.002 & \pm 0.0 & \pm 0 & \pm 0.002 \\
\hline \multirow[t]{3}{*}{ 1874-2009 } & \pm 0.005 & \pm 0.072 & \pm 0.001 & \pm 0.003 & \pm 0.050 & \pm 0 \\
\hline & \multicolumn{6}{|c|}{ Southern hemisphere } \\
\hline & \multicolumn{4}{|c|}{ First appearance of spots } & All spots & \\
\hline time & $E_{\Omega_{0}}$ & $E_{B}$ & $E_{\Omega_{1}}$ & $E_{\Omega}$ & $\overline{E_{B}}$ & $\overline{E_{\Omega}}$ \\
\hline 1 cycle & $=0.021$ & \pm 0.237 & \pm 0.005 & \pm 0.016 & \pm 0.192 & \pm 0.003 \\
\hline 3-cycle & \pm 0.016 & \pm 0.200 & \pm 0.003 & \pm 0.015 & \pm 0.192 & \pm 0.002 \\
\hline 6-cycle & \pm 0.013 & \pm 0.167 & \pm 0.002 & \pm 0.011 & \pm 0.156 & \pm 0.002 \\
\hline $1878-1996$ & \pm 0.008 & \pm 0.113 & \pm 0.002 & \pm 0.004 & \pm 0.067 & \pm 0.001 \\
\hline 1874-2009 & \pm 0.005 & \pm 0.073 & \pm 0.001 & \pm 0.004 & \pm 0.057 & \pm 0.001 \\
\hline
\end{tabular}

\section{Non-axisymmetry}

We define the measure of non-axisymmetry $\Gamma$ in the same way as earlier

$\Gamma=\frac{W_{1}-W_{2}}{W_{1}+W_{2}}$

where $W_{1}$ and $W_{2}$ denote the normalized area of sunspots that appeared within $\left(W_{1}\right)$ or outside $\left(W_{2}\right)$ the two active longitudes, which are taken here as the two bands

$W_{1}=\sum_{k, i} W_{i k}$, if $\left(\left|\lambda_{\mathrm{ik}}-\Lambda_{\mathrm{ik}}\right|<45^{\circ}\right.$ or $\left.360^{\circ}-\left|\lambda_{\mathrm{ik}}-\Lambda_{\mathrm{ik}}\right|<45^{\circ}\right)$

$W_{2}=\sum_{k, i} W_{i k}, \operatorname{if}\left(\left|\lambda_{\mathrm{ik}}-\Lambda_{\mathrm{ik}}\right|>45^{\circ}\right.$ and $\left.360^{\circ}-\left|\lambda_{\mathrm{ik}}-\Lambda_{\mathrm{ik}}\right|>45^{\circ}\right)$
We have plotted the values of $\Gamma$ for the individual cycles and the three-cycle periods in Fig. 6. One can see that there are large differences between the non-axisymmetries of the individual cycles. The largest non-axisymmetry for both hemispheres is found during cycle 16 . For the northern hemisphere the nonaxisymmetry during this cycle is significantly larger than during any other cycle. We note that this cycle marked the beginning of the rise in solar activity that culminated with the Modern Grand Maximum of the last century. Non-axisymmetry is smallest in both hemispheres during cycle 19, the record cycle according to sunspot activity. This result agrees with Vernova et al. (2002), who noted that the excessive sunspot activity during cycle 19 was longitudinally symmetric, while some other cycles were stronger in longitudinally ordered sunspot activity. 

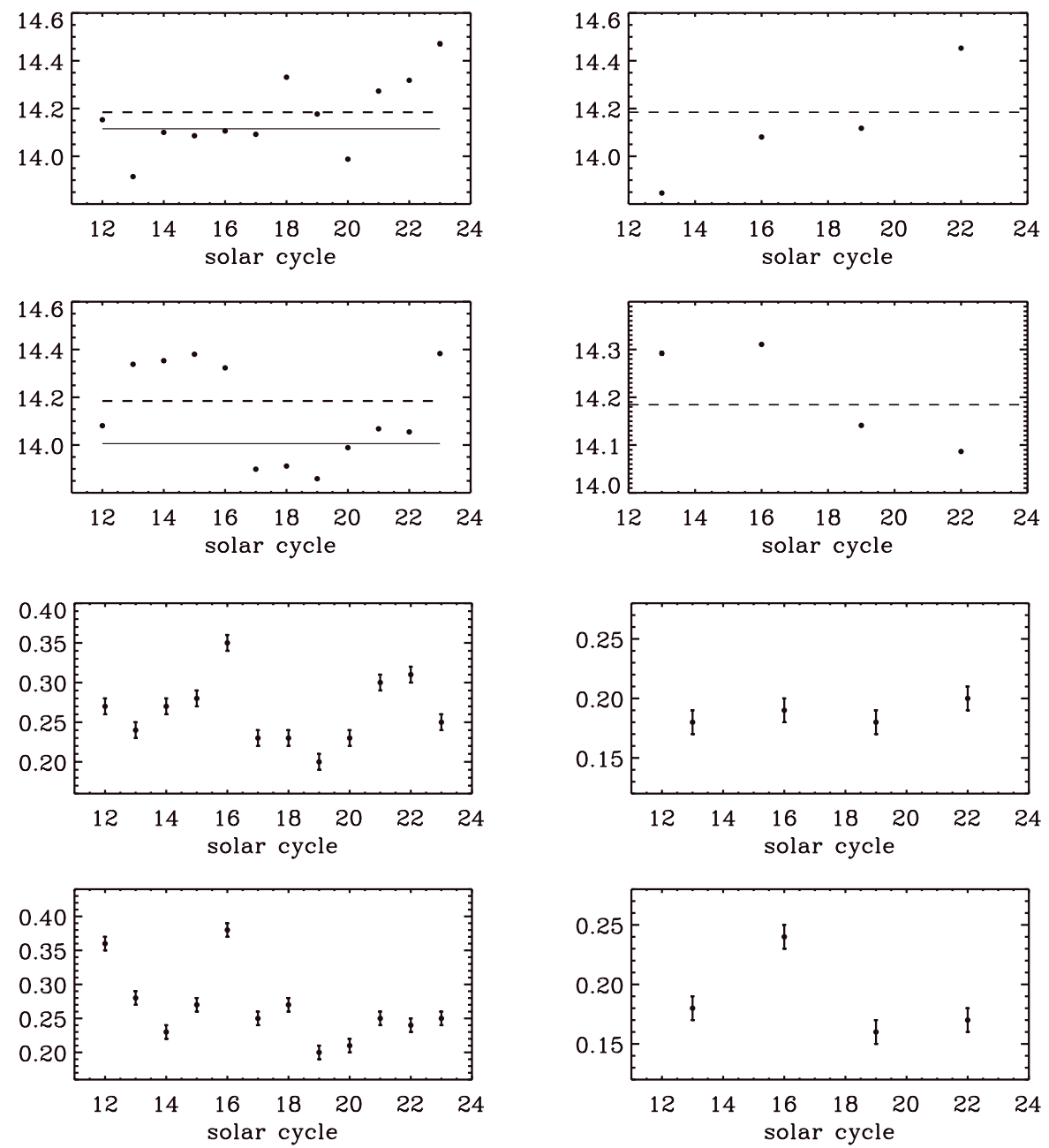

Fig. 5. Values of $\Omega_{17}$ using all sunspots for (left panels) individual cycles and (right panels) three-cycle intervals. The upper (lower) panels are for the northern (southern) hemisphere. The horizontal lines depict the best-fit value of $\Omega_{17}$ for the whole interval.
While the non-axisymmetry does not depict any clear trend or oscillation pattern, the non-axisymmetries of the two hemispheres seem to roughly follow each other except during the last few years. This is also seen in the three-cycle averages of Fig. 6. Furthermore, in Table 6 we have given the average values of the non-axisymmetry parameters for the different time lengths. The non-axisymmetry slowly decreases as the time length increases. This decrease in non-axisymmetry with increasing time interval most likely results from the long-term variation in solar rotation. Table 6 shows that non-axisymmetries for all sunspots are slightly but consistently higher than for the first appearances of sunspots. This suggests that strong, long-living sunspots occur and remain preferentially around the two active longitudes.

\section{Discussion}

Let us first compare the present values of the rotation parameters with those obtained earlier for sunspots using the original, slightly less correct treatment of the differentially rotating coordinate system of active longitudes. Usoskin et al. (2005) used the first appearance of sunspot groups and found the best-fit values of $\Omega_{0}=14.33 \pm 0.01$ (deg/day) and $B=3.40 \pm 0.03$ (deg/day) for the northern hemisphere and $\Omega_{0}=14.31 \pm 0.01(\mathrm{deg} / \mathrm{day})$ and $B=3.39 \pm 0.02$ (deg/day) for the southern hemisphere in 18781996. The corresponding values obtained here (see Table 4$)$ are $\Omega_{0}=14.393 \pm 0.008$ (deg/day) and $B=3.309 \pm 0.116$ (deg/day) for the northern hemisphere, and $\Omega_{0}=14.435 \pm 0.008$ (deg/day) and $B=4.931 \pm 0.113$ (deg/day) for the southern hemisphere.
Accordingly, the two values for $\Omega_{0}$ and the value of $B$ for the southern hemisphere obtained here are significantly larger than found earlier (Usoskin et al. 2005). The main difference between the two methods seems to be so that the best-fit solution now tends to emphasize the hemispheric difference, especially in the latitudinal variation parameter $B$, more strongly than earlier. This contradicts the result of Heristchi \& Mouradian (2009), who found no north-south asymmetry in solar rotation. Large $B$ values for both hemispheres strongly suggest that sunspot groups follow the differential rotation law, which differs from the conclusion of Balthasar (2007).

The present method leads to a larger asymmetry in the southern hemisphere than found earlier. The values of the $\Gamma$ parameter obtained now (see Table 6$)$ for the northern $(\Gamma=0.110)$ and southern hemisphere $(\Gamma=0.109)$ in $1878-1996$ are roughly equal, while the asymmetry in the north $(\Gamma=0.11)$ obtained by Usoskin et al. (2005) was the same as here but larger than in the south $(\Gamma=0.09)$. This suggests that the rotation of the southern hemisphere may be more complicated or variable than in the northern hemisphere, and that the present improved method is indeed more accurate in treating such variable rotation of active longitudes. We note also that the non-axisymmetry parameters for the first appearances of sunspots in the last three solar cycles obtained here ( 0.17 for north and 0.13 for south, see Fig. 6$)$ are significantly higher than those found by the earlier method $(0.12$ for north and 0.09 for south; Zhang et al. 2007b), giving further evidence for the superiority of the present method. 
Table 6. Average value of non-axisymmetry $\Gamma$ for different time intervals.

\begin{tabular}{lcccc}
\hline \hline & \multicolumn{2}{c}{ Northern hemisphere } & \multicolumn{2}{c}{ Southern hemisphere } \\
& First appearance of spots & All spots & First appearance of spots & All spots \\
\hline time & $\Gamma$ & $\Gamma$ & $\Gamma$ & $\Gamma$ \\
1 cycle & 0.223 & 0.266 & 0.233 & 0.263 \\
3-cycle & 0.153 & 0.163 & 0.155 & 0.188 \\
6-cycle & 0.120 & 0.140 & 0.115 & 0.125 \\
1878-1996 & 0.110 & 0.112 & 0.109 & 0.119 \\
1874-2009 & 0.108 & 0.092 & 0.092 & 0.103 \\
\hline
\end{tabular}

In the present method, the values of $\Omega_{0}$ are determined far more accurately than by the earlier method, but $B$ is determined less accurately. Although no exact values for individual cycles were given by Usoskin et al. (2005), one can see in their Fig. 5 that the range of $\Omega_{0}$ and $B$ for the northern hemisphere of the individual cycles varied roughly between 13.65 and 14.7 , and between 1.6 and 4.1, respectively, while here they were (see Table 1) roughly between 14.2 and 14.6, and between 0.4 and 4.3. However, despite these differences in the best-fit values of the two rotation parameters, the rotation rates at the maximum sunspot latitude are quite similar here $\left(\Omega_{17}=14.119 \pm 0.003\right.$ for north and $\Omega_{17}=14.008 \pm 0.002$ for south $)$ and earlier $\left(\Omega_{17}=\right.$ 14.04 for north and $\Omega_{17}=14.02$ for south). Thus we can verify the earlier observation that, over the whole interval, the mean rotation in the southern hemisphere is slower than in the north. The significance of this difference is considerably larger than found earlier. Pelt et al. (2007) also found that the average rotation was slower in the southern hemisphere.

As mentioned above, we used the improved method to study the differential rotation of active longitudes during the last three solar cycles using solar X-ray flares (Zhang et al. 2011). We found there that the new method increased the amount of nonaxisymmetry considerably and made the rotation parameters for three flare classes agree with each other. For instance, the for Cclass flares $\Omega_{0}=14.59 \pm 0.04$ and $B=1.43 \pm 0.50$ for the northern hemisphere and $\Omega_{0}=14.39 \pm 0.04$ and $B=2.80 \pm 0.50$ for the southern hemisphere. Comparing these with the present results depicted in Table 2, one finds excellent agreement for both parameters. The results for the two solar variables for the mean rotation rate at the latitude of maximum sunspot production also agree fairly closely, being $\Omega_{17}=14.453 \pm 0.003 / 14.468 \pm 0.002$ for north and $\Omega_{17}=14.087 \pm 0.001 / 14.151 \pm 0.002$ for south for sunspots versus C-class flares. These results verify with exceedingly high statistical significance that the two solar hemispheres rotated, on an average, considerably differently during the last three solar cycles, the northern hemisphere much faster, the southern hemisphere slightly lower than the Carrington rotation rate.

Finally, we would like to note on the long-term evolution of the rotation parameters. As seen in Fig. 3, the $\Omega_{0}$ values for the individual cycles depict relatively large and statistically highly significant temporal variations. Moreover, the long-term variations in $\Omega_{0}$ are quite different in the two hemispheres. In the northern hemisphere, there is indication of a long-term increasing trend, upon which a multi-decadal oscillation is superimposed. In the southern hemisphere, there may be a small decreasing trend, which may be superimposed on a century-scale oscillation. Interestingly, the long-term variations in $\Omega_{0}$ (both trends and oscillations) are roughly opposite in the northern and southern hemispheres. The $B$ values also depict large long-term variability (see Fig. 4), but there does not seem to be any systematic long-term development in either hemisphere, either in trend or oscillation. However, as for $\Omega_{0}$, the $B$ values depict roughly opposite long-term evolution in the two hemispheres, except during the two recent cycles.

We note that the long-term evolution of $\Omega_{17}$ (see Fig. 5) is quite similar to that of $\Omega_{0}$ despite the rather unsystematic longterm change in the $B$ parameter. This supports the view that the obtained results for the long-term evolution are indeed reliable and significant. Compared to $\Omega_{0}$, the long-term evolution of $\Omega_{17}$ for individual cycles seems to emphasize the increasing trend in the northern hemisphere, suppressing the amplitude of oscillation around the trend, while the centennial oscillation appears more systematically in the southern hemisphere in $\Omega_{17}$ than in $\Omega_{0}$. The three-cycle values also underline the opposite temporal evolutions in the two hemispheres with increasing rotation rate in the north and decreasing rotation in the south.

Earlier studies have already noted that the rotation parameters depict long-term trends and long-term oscillations (see, e.g., Makarov et al. 1997; Kitchatinov et al. 1999; Javaraiah et al. 2005; Brajsa et al. 2006, and references therein). For instance, Javaraiah et al. (2005) found that the $\Omega_{0}$ parameter depicts a fairly systematic decline during cycles 12 to 23 from roughly $\Omega_{0}=14.55 \mathrm{deg} / \mathrm{day}$ to $\Omega_{0}=14.45 \mathrm{deg} / \mathrm{day}$, with some evidence of weak century-scale oscillation superimposed. The $B$ parameter was also found to have a small decreasing trend from about $B=2.7$ to about $B=2.4$, but the evolution of $B$ was dominated by a roughly 80 -year oscillation with an amplitude of $\pm 0.3 \mathrm{deg} /$ day. However, these results cannot be directly compared with the long-term evolution of the best-fit values of the two rotation parameters found in this study since Javaraiah et al. (2005) did not separately consider the two hemispheres.

As noted above, the largest non-axisymmetry for both hemispheres is found for cycle 16, which started the rise of solar activity in the 20th century. On the other hand, the smallest asymmetry is found for cycle 19, which was the most active cycle, starting the decrease in sunspot activity thereafter. Although the values of $\Omega_{0}$ or $\Omega_{17}$ were considerably larger for cycle 16 than cycle 19 in both hemispheres, they were not exceptional or extremum values for either of these two cycles. Accordingly, the relation between solar rotation and solar activity seems to be rather complicated.

Berdyugina et al. (2006) and Usoskin et al. (2007) summarized the dynamo modes related to the occurrence of preferred longitudes in solar and stellar activity and discussed the interpretation of the existence of active longitudes within several solar dynamo related and other scenarios (see also Ruzmaikin 1998; Rädler et al. 1990; Moss 1999, 2004; Mason et al. 2002; Fluri \& Berdyugina 2005; Bassom et al. 2005; Korhonen \& Elstner 2005). Berdyugina et al. (2006) noted that in most dynamo models the generated structures are expected to rotate quasirigidly, while the migration of active longitudes could be caused by a stroboscopic effect. Nevertheless, the significantly varying rate of asymmetry and rotation from one cycle to another, as well as the significant hemispheric differences provide observational constraints that need to be taken into account in dynamo modeling. 


\section{Conclusions}

Using a refined version of the dynamic, differentially rotating coordinate system, we have determined the optimal values of the two rotation parameters $\Omega_{0}$ and $B$ (see Eq. (1)) and the average rotation rate $\Omega_{17}$ for all sunspot groups and the first appearances of sunspots in the two hemispheres separately. We have also calculated these parameters for different lengths of time. We list below our main results and findings:

1. We found the following parameter values for, e.g., all sunspots over the whole 136-year time interval: $\Omega_{0}=$ $14.411 \pm 0.003$ (deg/day) and $B=3.455 \pm 0.050$ (deg/day) for the northern hemisphere and $\Omega_{0}=14.401 \pm 0.004(\mathrm{deg} / \mathrm{day})$ and $B=4.652 \pm 0.083$ (deg/day) for the southern hemisphere. Typically, the refined method yielded somewhat larger values for $\Omega_{0}$ than the earlier method (Usoskin et al. 2005).

2. The values of $\Omega_{0}$ were determined much more accurately but $B$ slightly less accurately than earlier (Usoskin et al. 2005). However, the average rotation rates $\Omega_{17}$ at the maximum sunspot latitude of $17^{\circ}$ in $1878-1996$ were found to be almost similar to earlier values.

3. The refined method yielded closely similar asymmetries in the two hemispheres, and a larger asymmetry in the southern hemisphere in 1878-1996 than earlier (Usoskin et al. 2005). In addition, the non-axisymmetry of the first appearances of sunspots in the last three solar cycles are significantly higher here than those found by the earlier method (Zhang et al. 2007b). This gives further evidence of the credibility and importance of the improvement of the method.

4. The optimal values of the two differential rotation parameters obtained here for all sunspots are in close agreement with those for first appearance spots in both hemispheres and all time intervals.

5. The two rotation parameters for sunspots during the last three solar cycles agree closely with the parameters obtained for different classes of X-ray flares using the same refined method. To our knowledge, such good agreement between rotation parameters of sunspots and flares in both hemispheres is unique.

6. Non-axisymmetry in sunspots during the last three solar cycles was somewhat smaller than in X-ray flares. Also, nonaxisymmetry in all sunspots was systematically larger than for the first appearances of spots. These results support the view that strong and persistent forms of solar activity follow more closely to the two active longitudes.

7. The largest non-axisymmetry for both hemispheres is found during cycle 16 . It is interesting to note that this cycle started the rise of solar activity to the Modern Grand Maximum of the last century. Non-axisymmetry is smallest in both hemispheres during cycle 19 , the record cycle according to sunspot activity.

8. We found that the errors in optimal parameters decreased slowly but systematically with increasing time interval, implying that there is a fairly good temporal consistency in the rotation parameters.

9. The refined method emphasizes the hemispheric differences in the two parameters. Over the whole interval, the mean rotation in the southern hemisphere is slower than in the north. The significance of this difference is considerably larger than earlier. There is overwhelming agreement from sunspots and flares that the northern hemisphere rotated much faster than and the southern hemisphere slightly slower than the Carrington rotation rate during the last three solar cycles.
10. There is significant temporal variability in the two rotation parameters over the 136-year interval. In the northern hemisphere, $\Omega_{0}$ and $\Omega_{17}$ follow long-term increasing trends, upon which a weak multi-decadal oscillation may be superimposed. In the southern hemisphere, $\Omega_{0}$ and $\Omega_{17}$ exhibit decreasing trends, superimposed on a significant century-scale oscillation. Interestingly, the long-term variations in $\Omega_{0}$ and $\Omega_{17}$ (both trends and oscillations) are roughly opposite in the northern and southern hemispheres.

Acknowledgements. The research leading to these results has received funding from the European Commission's Seventh Framework Programme (FP7/2007-2013) under the grant agreement No. 218816 (SOTERIA project, www.soteria-space.eu). We also acknowledge the financial support by the Academy of Finland to the HISSI research project No. 128189. This work is jointly supported by the National Basic Research Program of China (Program 973) through grant 2006CB806307, the National Natural Science Foundation of China through grants 10673017, 10733020, 10973020 and 40890161, and Projects KJCX2-YW-T04 and KGCX3-SYW-403-10 of the Chinese Academy of Sciences.

\section{References}

Bai, T. 1988, ApJ, 328, 860

Bai, T. 2003, ApJ, 591, 406

Balthasar, H. 2007, A\&A, 471, 281

Bassom, A., Kuzanyan, K., Soward, A. M., \& Sokoloff, D. 2005, Geophys. Astrophys. Fluid Dyn., 99, 309

Benevolenskaya, E. E., Hoeksema, J. T., Kosovichev, A. G., \& Scherrer, P. H. 1999, ApJ, 517, L163

Berdyugina, S. V., \& Usoskin, I. G. 2003, A\&A, 405, 1121

Berdyugina, S. V., Moss, D., Sokoloff, D., \& Usoskin, I. G. 2006, A\&A, 445, 703

Brajsa, R., Rudjak, D., \& Wöhl, H. 2006, Sol. Phys., 237, 365

Bumba, V., Garcia, A., \& Klvana, M. 2000, Sol. Phys., 196, 403

Chidambara, A. 1932, MNRAS, 93, 150

Fluri, D. M., \& Berdyugina, S. V. 2005, in 22nd NSO Workshop Large Scale Structures and their Role in Solar Activity, ed. K. Sankarasubramanian, M. Penn, \& A. Pevtsov, ASP Conf. Ser., 346, 167

Hathaway, D. H., Wilson, R. M., \& Reichmann, E. J. 2002, Sol. Phys., 211, 357

Heristchi, D., \& Mouradian, Z. 2009, A\&A, 497, 835

Javaraiah, J., Bertello, L., \& Ulrich, R.K. 2005, Sol. Phys., 232, 25

Juckett, D. A. 2006, Sol. Phys., 237, 351

Kitchatinov, L. L., Pipin, V. V., Makarov, V. I., \& Tlatov, A. G. 1999, Sol. Phys., 189,227

Korhonen, H., \& Elstner, D. 2005, A\&A, 440, 1161

Losh, H. M. 1939, Publications of the Observatory of the University of Michigan; v. 7, No. 5 (Ann Arbor: University of Michigan Press), 127

Makarov, V. I., Tlatov, A. G., \& Callebaut, D. K. 1997, Sol. Phys. 170, 373

Mason, J., Hughes, D. W., \& Tobias, S. M. 2002, A\&A, 580, L89

Moss, D. 1999, MNRAS, 306, 300

Moss, D. 2004, MNRAS, 352, L17

Mursula, K., \& Hiltula, T. 2004, Sol. Phys., 224, 133

Pelt, J., Korpi, M. J., \& Tuominen, I. 2010, A\&A, 513, A48

Rädler, K.-H., Weidemann, E., Meinel, R., Brandenbury, A., \& Tuominen, I. 1990, A\&A, 239, 413

Ruzmaikin, A. A. 1998, Sol. Phys., 181, 1

Ruzmaikin, A., Feynman, J., Neugebauer, M., \& Smith, E. J. 2001, J. Geophys. Res., 106, 8363

Solanki, S. K. 2003, A\&A, 11, 153

Takalo, J., \& Mursula, K. 2002, Geophys. Res. Lett., 29, 1317

Usoskin, I. G., Berdyugina, S. V., \& Poutanen, J. 2005, A\&A, 441, 347

Usoskin, I. G., Berdyugina, S. V., Moss, D., \& Sokoloff, D. D. 2007, Adv. Space Res., 40, 951

Vernova, E. S., Mursula, K., Tyasto M. I., \& Baranov, D. G. 2002, Sol. Phys., 205, 371

Zhang, L. Y., Cui, Y. M., He, Y. L., et al. 2007a, Adv. Space Res., 40, 970

Zhang, L. Y., Wang, H. N., Du, Z. L., Cui, Y. M., \& He, H. 2007b, A\&A, 471, 711

Zhang, L. Y., Mursula, K., Usoskin, I., \& Wang, H. N. 2011, J. Atmosph. SolarTerr. Phys., 73, 258 